\title{
Some key Techniques Research Based on Five-axis Matching of The Integral Impeller
}

\author{
Chun-guang $\mathrm{Gu}^{\mathrm{a}}$, Zhu-ming $\mathrm{Cao}^{\mathrm{b}}$ and Hong-mei Sun ${ }^{\mathrm{c}}$ \\ Beijing Polytechnic,Beijing 100176, China; \\ a guchunguang@263.net, bczm654@126.com, 'c sunhongmei3412@sohu.com
}

Keywords: Integral Impeller,5-axis Machining,Key Technologies,Research,Tool Path,Technology \& Equipment.

\begin{abstract}
Integral impeller is the key component of aeroengine, which reflects the development level of a country's equipment manufacturing industry. The function, technical requirements and processing difficulty were introduced in the paper. The machining processes of a axial-flow impeller was described. The core technologies, including processes, fixtures, tools, cutter path design, simulation and the actual processing, were analyzed. It is proved that the process and the tool path strategy were very successful, and the integral impeller which was produced has satisfied the design and accuracy requirements. Finally, this paper presents the study focus of the impeller and the problem to be solved.
\end{abstract}

\section{Introduction}

Aviation industry is a symbol of the country's comprehensive national strength; the core technology of the aviation industry is engine technology; aero-engine has become a national science and technology level, an important symbol of military power and comprehensive national strength. According to statistics, $80 \%$ of aviation engine components are impellers and blades. If the core is the engine of the aircraft, then the core of the engine is the impeller blades. Therefore, the impeller blades processing technology research, the development of the aviation industry has an important significance. Overall impeller operation mode by airflow into axial flow impeller and centrifugal impeller. When the axial flow impeller work, air flow direction parallel to the axial direction, primarily to provide forward motion of the aircraft power; while a centrifugal impeller work, away from the direction of the air flow in the axial direction, the main provider of aircraft to rise to power .

This article is to introduce the whole aviation axial flow impeller machining, as shown in Fig. The overall impeller basic parameters and technical requirements are: aluminum twisted blades, open integrated impeller; maximum diameter of $200 \mathrm{~mm}$, the number of blades of 16 , hanging long blade $45 \mathrm{~mm}$, blade width $50 \mathrm{~mm}$, maximum thickness of the blade $1.6 \mathrm{~mm}$, the minimum thickness of $0.6 \mathrm{~mm}$; curved blade surfaces and the hub surface quality Ra1.6, leaf shape accuracy error of \pm $0.03 \mathrm{~mm}$.

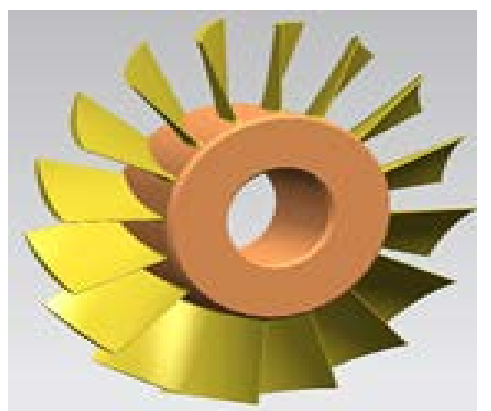

Fig1. Axial flow impeller overall

To ensure the overall aerodynamic performance of the impeller to achieve the requirements of aeroengine blade impeller on the whole are generally composed of complex surfaces. The complex is the result of a three-dimensional twisted surfaces formed geometric accuracy requirements are high. At the same time due to the overall impeller in the harsh environment of high temperature and high 
pressure, so the choice of materials are basically difficult to machine materials, machining integral impeller therefore requires not only high-end five-axis machine tools, but also efficient CAD / CAM software to design the cutter path. At the same time carrying out multi-axis machining integral impeller, since the tool spindle table and need to rotate so that the spindle and table collision probability increases, which requires special fixture design will be uplifted blank to avoid a collision phenomenon. Difficulties also integral impeller processing further comprising: a gap between adjacent blades small, relatively long blades, low stiffness, are thin-walled parts, the process prone to distortion; the adjacent blades result in minimal space clearance angle processing When the tool diameter is smaller, the tool is easy to break; leaf distortion curvature, prone to interference when processing large processing difficulty. In summary, the aviation integral impeller processing involves machines, tools, materials, technology and other aspects of technology, we can say the overall impeller processing technology is one important indicator of a country's equipment manufacturing industry.

\section{CNC machining integral impeller}

Processing Method.CNC machining integral impeller milling methods are divided into point method, side milling method and three milling interpolation method. Point ball milling method is to use a knife blade by blade progressive flow direction passes, gradually machined blade surfaces. Processing site for the ball, the contact point contact manner, this processing method can better guarantee of curved surfaces and high precision, but the relatively low efficiency. The second category is a side milling method, which uses a cylindrical or conical milling swarf milling cutter blade surfaces, it is mainly used for direct bus impeller machining. Machining parts for the tool side of the blade; contact manner in line contact; characterized this processing method can get better surface quality, high efficiency, but the arbor complexity of the algorithm. Plunge milling method is the time from the top down has been milled to an impeller blade root or runner surface. And by lateral translation tool feed machining complex surface geometry. Plunge milling method has high processing efficiency, processing time is short, more suitable for the removal of such a large amount of integral impeller roughing complex parts. For this plunging, plug cutter track layout has been the difficulty of its processing. This article describes both impeller machining point milling method used, is also used in side milling method.

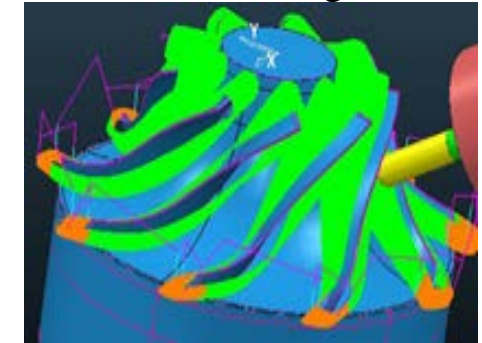

(A)point milling method

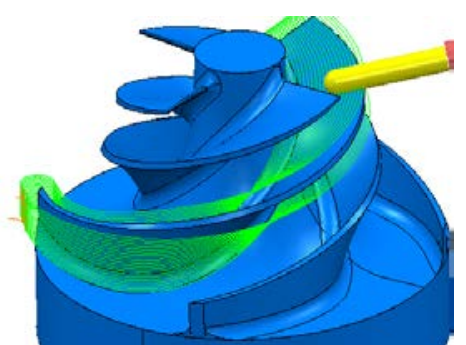

(B) side milling method

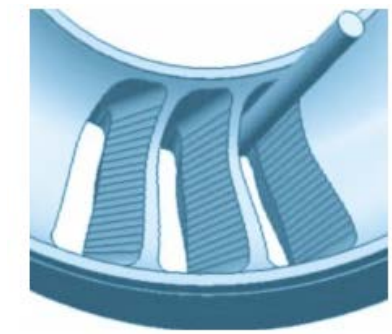

(C) plunge milling method

Fig.2impellermachiningstrategy

Tool selection.Tooling overall impeller used are cylindrical knife, knife cone, drum cutter, special type of knives. In the opening process should be used wherever possible roughing a large diameter tip arc straight shank end mill to improve efficiency; during finishing, due to a smaller gap between the blades, blade roots deeper, mostly chosen Cone shank end mill to improve tool rigidity, while the CAM software should be finishing tool shank taper value is set larger than the actual, to avoid the phenomenon of under-cut or over-cut finishing. In order to improve processing efficiency and ensure that the tool rigidity, usually obtained from the analysis with CAD software is the impeller blade pitch process, and in order to ensure the semi-finishing allowance and the arbor swing angle reserve space, according to Figure 3 estimated tool diameter of formula (h: blades length). Calculated according to the formula, the impeller roughing article describes the selection $\varnothing 12$ ball cutter, finishing the selection taper shank of ball milling Ø4. 


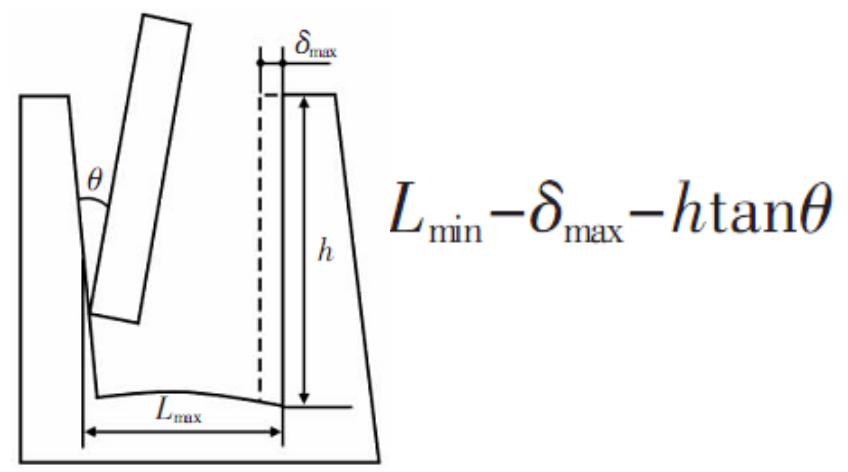

Fig. 3 overall selection impeller machining tools

\section{Tooling design}

Free curved impeller blades described in this article is the result of a three-dimensional form of twisted, so need to use the five-axis machining CNC machining. This article describes the use Delcam software company PowerMill cutter path design, and generate NC post-processing program, the final completion of the processing in Beijing Vocational College of Electronic Science and Technology Training Center use on DMU60 CNC five-axis machining centers. Machining the part using a roughing and two finishing (respectively vane hub finishing and finishing), the process as shown in Table 1.

Table1. Processing Technology

\begin{tabular}{c|c|c|c|c}
\hline No. & $\begin{array}{c}\text { processing } \\
\text { content }\end{array}$ & processing strategy (POWERMILL) & Tool & Notes \\
\hline 1 & Coarse & Leaf disc area clear & $\varnothing 12$ ball knife & \\
\hline 2 & Finishing 1 & Finishing blade & $\varnothing 12$ ball knife & \\
\hline 3 & Finishing 2 & Hub finishing & $\begin{array}{c}\varnothing 10 \text { taper shank } \\
\text { ball-cutter }\end{array}$ & \\
\hline
\end{tabular}

Special fixtures in the part of the five-axis machining process, since the machine $\mathrm{B}, \mathrm{C}$ axis must rotate the handle easily interfere with the jig, so you need to be uplifted clamping position of the workpiece, which need to use an impeller machining the parts used in the jig shown in Figure 4 . The main technical features are: $\varnothing 60 \times 190$ cylindrical facilitate universal clamping jaw self-centering chuck, alignment, installed in center of the table, the full application of multi-axis machine travel; $\varnothing 50$ / Ø110 at the shoulder, and 2 undercut $\times 0.5$, and applied to be accurately positioned machining parts; $4 \times \varnothing 8.5$ hole, and applied to the locking bolt work (applied to the centrifugal impeller clamping); pin hole Ø6 and applied workpiece positioning; M30 thread for locking a workpiece (for axial flow impeller clamping); fixture, with parts of the roughness, concentricity and vertical degree higher, ensure clamping accuracy.

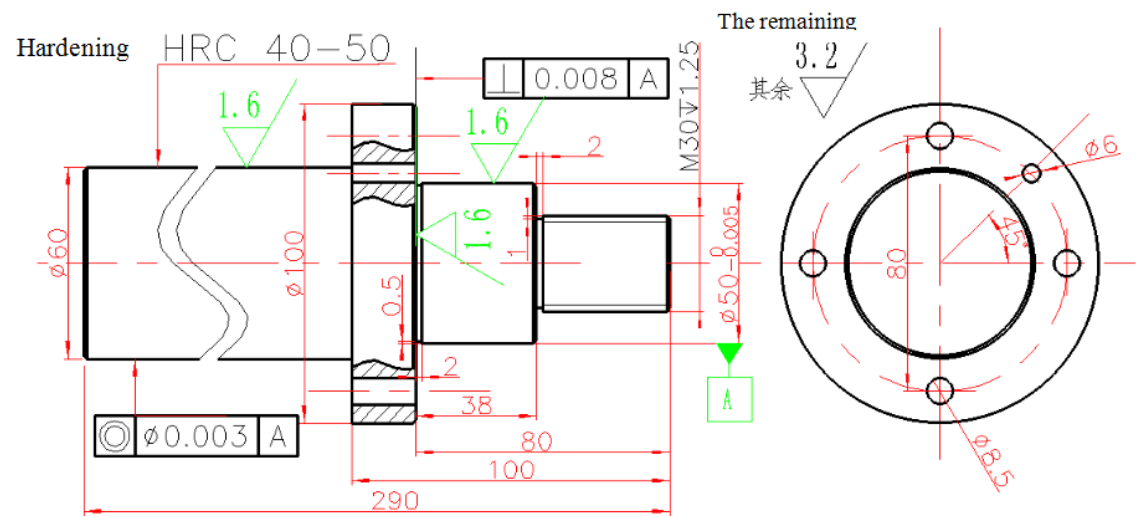

Fig. 4 Fixture Design 


\section{Cutter path design}

Aviation integral impeller processing basically belong axis CNC machining, key according to the actual machining situation, the application of CAD / CAM software to design the cutter path. Today, five-axis can be programmed software UG, hyperMILL, cimatron, powermill, caxa manufacturing engineers, etc., which due powermill software is a powerful, easy to operate and so, in the domestic market share is increased every year. This article describes the use of integral impeller powermill software "leaf disc" module to prepare axis cutter path.

Impeller machining strategy operating powermill belongs parameterization, the first to build two secondary surfaces, which are wheels (auxiliary surface), sets (auxiliary surface), and then left-wing blade, wing blades, a hub (auxiliary surface), sets (auxiliary surface) were stratified set, then during the leaf discs area clear, blade finishing, finishing three hub strategy design, it will be filled with the appropriate characteristics, as shown in Figure 5-a, and then in the "ignore" option be sure the box will "set" surface set to "ignore" feature, and then the tool axis limits, toolpath connection settings (security height of the cylinder can be set to improve the processing efficiency), and finally gouge and collision check, can As shown generate integral impeller machining tool path shown 5-b.

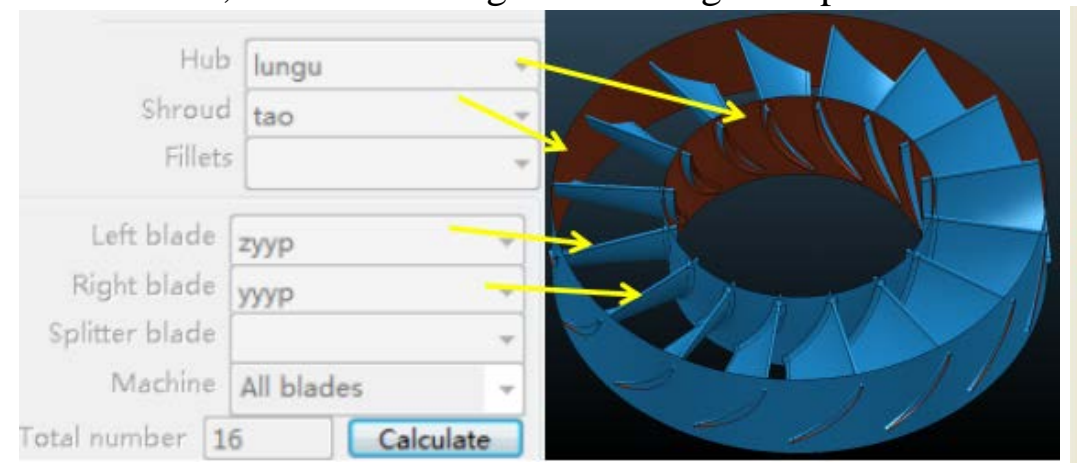

(A) Construction of auxiliary plane Fig. 5 Overall impeller cutter path settings

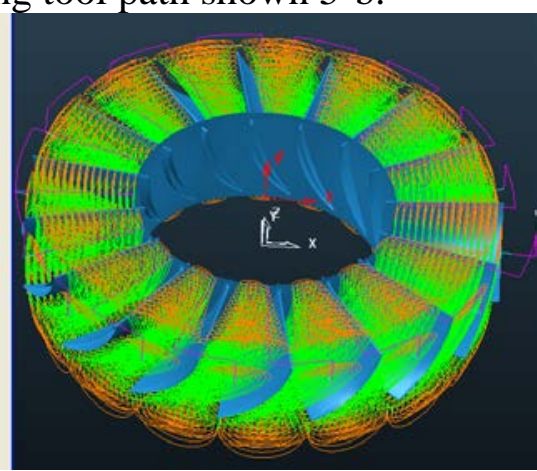

(B) cutter path

\section{integral impeller processing}

Simulation Machining.To ensure safe and reliable five-axis machining is required before the actual machining, tooling based on the actual processing time will be simulated processing. This selection vericut simulation software simulation process. After the cutter path POWERMILL before processing software design needs to be set to handle a machine identification NC program, then build a simulation process simulation software platform, including machine tools, systems, fixtures, tools, etc., then the simulation process can be checked out whether there is a collision or under-cutting, over-cutting phenomenon. In VERICUT simulation process, when using five-axis machine simulation with a B-axis, zero configuration program to select from the component (B-axis) to the component (stock), so as to successfully complete the simulation work on the knife. Upon examination, the use of the tooling and machining strategies can be successfully processed into integral impeller, as shown in Figure 6 effect of each step after processing.

Actual processing. When the actual processing, using the device is DMU 60 MONOBLOCK five-axis machining centers, CNC system HEIDENHAIN ITNC530, you first need to convert the file into a ".h" format, and then established between the software, the computer and the five-axis machining center communication links (Information is to establish a TCP / IP protocol), via NC program transfer software CNC system HEIDENHAIN ITNC530 the NC program into the NC system. S roughing process is $4500 \mathrm{r} / \mathrm{min}, \mathrm{F}$ is $1000 \mathrm{~mm} / \mathrm{min}$; when finishing $\mathrm{S}$ is $8000 \mathrm{r} / \mathrm{min}, \mathrm{F}$ is $2000 \mathrm{~mm} / \mathrm{min}$, when machining coolant injection using the top, in-process blank, equipment and results are shown in 7. 


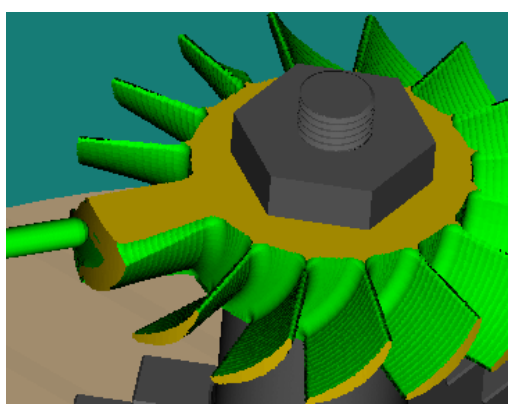

(A) open crude

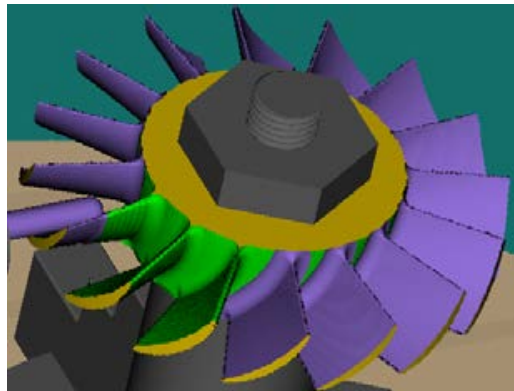

(B) finishing blades

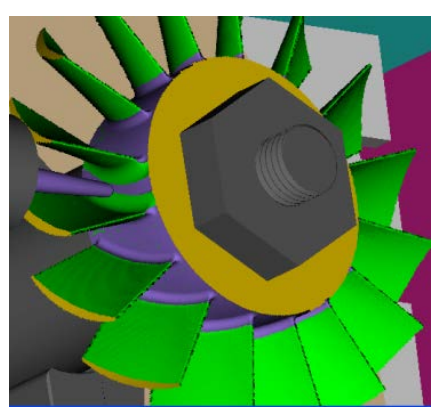

(C) the hub finishing

Fig. 6 integral impeller machining simulation

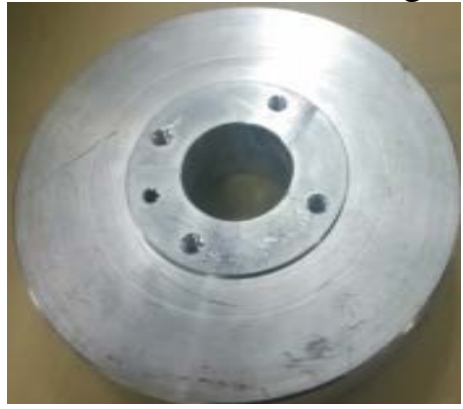

(A) blank

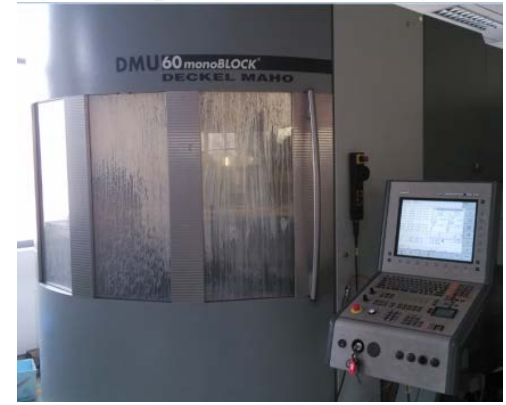

(B) processing equipment

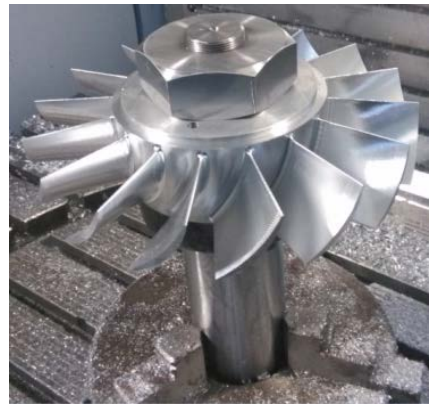

(C) processing results

Fig. 7 actual machining integral impeller

Actual fixture during processing by clamping jaw self-centering chuck, workpiece clamping at the same time try to table center, to ensure maximum stroke machine work implemented; to use dial indicator plus $\mathrm{C}$-axis mode knife, found $\mathrm{X}$ center $\mathrm{Y}$-axis, Z-axis positioner through the Z-axis of the knife; needed before processing tool length measured by the tool length measuring instrument and then enter the numerical control system; need to use as much as possible the process of cutting aluminum specific tool, plus cooling liquid, to avoid sticking knife phenomenon; in the case of tool length sufficient to shorten the length of the tool projecting possible. With this processing strategy and parameters, the successful completion of the processing of the specimen, the detection size and reach accuracy.

\section{Summary}

Aviation aeroengine integral impeller is the core component of the overall impeller processing technology reflects the level of development of a country's manufacturing process. This article describes a system of axial flow impeller aviation overall processing flow, including parts technical characteristics, processing methods, cutter path design, simulation and processing as well as the actual processing precautions; details of all aspects of the core technology.

Integral impeller processing needs more comprehensive modern manufacturing techniques, involving mathematical modeling, CAD / CAM technology, virtual manufacturing technology, machinery processing base and so on. Worth studying the overall direction of the impeller machining also many, such as connecting the tool path optimization, tool path algorithm, tool design, etc., can effectively improve the quality and efficiency of the processing parts. Impeller process by force is a dynamic process, the tool cutting force will result in the blades of the impeller deformation, resulting in reduced machining accuracy. So cutting force impact on the overall deformation of the impeller will be the focus of future research in this field.

\section{Reference}

[1] Zeng luxury Based integral impeller processing technology and NC programming UG NX6.0 of [J]. Coal Mine Machinery, 201110. 
[2] Chen Guangming research based process design principles and methods of CNC machining [J]. Manufacturing Automation, 200509.

[3] Wang Xiankui, Wang Renkang. Modern manufacturing technology and its development trend [J]. Modern Manufacturing Engineering, 200801.

[4] Guo Xionghua, Zucker recalls .PowerMill after a vehicle chassis front member check processing application and research [J] tools. Manufacturing Automation, 201007.

[5] Yang Xiaoqin, Tao Jianhua, early Liu, Zhu Mingying Developers electrode machining for NC automatic programming system [J]. Machine Tool \& Hydraulics, 201106.

[6] Zhao Yuxia, etc. Based on five-axis machine tools and machining impellers solid modeling [J]. Manufacturing Technology \& Machine, 201112.

First Author: Chun-guang Gu, male, born in 1981, master's degree, lecturer, research direction for multi-axis CNC machining technology.

Corresponding Author:Zhu-ming Cao, male, born in 1981, master's degree, lecturer, research direction for multi-axis CNC machining technology, has published more than 20 papers, guidance and participation of numerous CNC skills competition and winning.

Address: Beijing Economic and Technological Development Zone, a street Liangshuihe Nine (Beijing Polytechnic Institute of Mechanical Engineering), Postcode: 100176, Email: czm654@126.com, Tel: 13581913487. 